Yasuhiro Kazuki • Tokuyuki Shinohara

Kazuma Tomizuka • Motonobu Katoh • Atsuko Ohguma

Isao Ishida $\cdot$ Mitsuo Oshimura

\title{
Germline transmission of a transferred human chromosome 21 fragment in transchromosomal mice
}

Received: May 1, 2001 / Accepted: June 25, 2001

\begin{abstract}
We generated transchromosomal (Tc) mice containing a human chromosome 21 fragment (hCF21) using mouse embryonic stem (ES) cells with the transferred hCF21. Here we report breeding analyses that test the maintenance rate of the hCF21 in Tc mice of two different genetic backgrounds, MCH (ICR) and C57BL/6. Fluorescence in situ hybridization and polymerase chain reactionbased DNA analyses revealed that the structure of the hCF21 fragment including the CBR1, SIM2, HLCS, and $D 21 S 268$ markers, was approximately $5 \mathrm{Mb}$ in size, and was transmitted at least to the F3 generation. Though the retention rate of the hCF21 was variable among individual mice, for example, $21 \%-92 \%$ in brain and $10 \%-92 \%$ in tail fibroblasts, the $\mathrm{C} 57 \mathrm{BL} / 6$ background yielded a higher retention rate than did the MCH (ICR). These results suggest that the hCF21 could be maintained stably in Tc mice, depending on the genetic background. The panel of Tc mice will be a useful model to investigate the function of genes on the hCF21 fragment in various tissues through germinal transmission.
\end{abstract}

Key words Germline transmission - Chromosome 21 . Transchromosomal mouse · Human artificial chromosome . ES cells $\cdot$ Microcell-mediated chromosome transfer

\section{Introduction}

Trisomy 21 (Ts21) is the most frequent cause of congenital heart defects and the leading genetic cause of mental retardation in humans (Korenberg et al. 1994; Kola and Hertzog

Y. Kazuki $\cdot$ T. Shinohara $\cdot$ M. Katoh $\cdot$ M. Oshimura $(\square)$

Department of Molecular and Cell Genetics, School of Life Sciences, Faculty of Medicine, Tottori University and CREST (JST),

86 Nishimachi, Yonago, Tottori 683-8503, Japan

Tel. +81-859-34-8260; Fax +81-859-34-8134

e-mail: oshimura@grape.med.tottori-u.ac.jp

K. Tomizuka $\cdot$ A. Ohguma $\cdot$ I. Ishida

Pharmaceutical Research Laboratory, KIRIN Brewery Co., Ltd.,

Takasaki, Japan
1997). To investigate the gene dosage effects of an extra copy of human chromosome 21 (hChr 21) on various phenotypes, we recently generated transchromosomal (Tc) mice containing hChr 21 (Shinohara et al. 2001). Chimeric mice containing a substantial content of hChr 21 transmitted a part of the fragment, approximately $5 \mathrm{Mb}$ of hChr 21 , including the CBR1, SIM2, and HLCS genes, through the germline to $\mathrm{F} 1$ mice. However, the retention rate of the human chromosome 21 fragment (hCF21) in chimeric mice varied because the hCF segregated independently and tended to be lost randomly during development, which led to phenotypic variations. Previously, we reported that the stability of transferred hCF2 and hCF14 varied depending on the genetic background (Shinohara et al. 2000). In the present study, breeding analyses were performed to address the effect of the genetic background on the stability of hCF21 in offspring. In the Tc mice of F2 and F3 generations, we examined (1) the region of hCF21 that was retained, (2) the integrity of the hCF21 in tail fibroblasts, and (3) the stability of hCF21 in tail fibroblasts and cerebral cortex in different genetic backgrounds.

In the Tc mice, the hCF21 was structurally stable, with no cytogenetically detectable rearrangement or deletion. The level of retention of hCF21 was relatively higher in the C57BL/6 genetic background than in the $\mathrm{MCH}$ background. These results indicate that the centromere of the hCF21 functions in the mouse cells, and that different strains have genetic-based differences in their level of competence for maintaining $\mathrm{hCF}$.

\section{Materials and methods}

Animals

$\mathrm{MCH}$ (ICR) and C57BL/6N mice were purchased from Japan Crea (Tokyo, Japan). 
Production of progeny

Establishment of the embryonic stem (ES) cells carrying the hCF21 and production of the chimeric mice with the ES cells is described elsewhere (Shinohara et al. 2001). Briefly, mouse A9 cells containing a single human chromosome 21 were screened from a human monochromosomal hybrid library (Inoue et al. 2001). The human chromosome 21 was transferred from the A9 hybrid cells into the TT2F mouse ES cells via microcell-mediated chromosome transfer. Chimeric mice were produced by injection of the ES cells into eight-cell stage embryos of MCH (ICR) mice (Shinohara et al. 2001). Progeny was produced following the protocol described in Fig. 1a.

Polymerase chain reaction (PCR) analysis

Genomic DNA from tail fibroblasts were first screened for the presence of human DNA by Alu-PCR. PCR for analyzing the retained region of hChr 21 was performed using hChr 21-specific sequence tagged site (STS) markers as described elsewhere (Shinohara et al. 2001).

Fluorescence in situ hybridization (FISH) analysis

Preparation of chromosome samples and FISH analyses were performed by standard methods. The mice were killed by cervical dislocation, and the cerebral cortex was isolated and homogenized. The samples were incubated for $15 \mathrm{~min}$ in $0.075 \mathrm{M} \mathrm{KCl}$, fixed with methanol and acetic acid $(3: 1)$, and then slides were prepared using standard methods. The probes were digoxigenin (Boehringer, Manheim, Germany)-labeled human COT-1 DNA (BRL, Rockville, MD, USA). Digoxigenin-labeled probes were detected with antidigoxigenin-rhodamine (Boehringer). The chromosomes were counter-stained with $4^{\prime}-6^{\prime}$-diamindino-2-phenylindole (Sigma, St. Louis, MO, USA).

Statistical analysis

The difference in retention rate of the hCF between the two genetic backgrounds was evaluated using a $t$-test.

\section{Results and discussion}

Production of progeny

Mice carrying the hCF21 were bred to the $\mathrm{MCH}$ (ICR) or C57BL/6 strains as summarized in Fig. 1a, and progeny was produced. First, a female chimeric mouse was bred with an $\mathrm{MCH}$ (ICR) male mouse. The resulting F1 generation was bred to either the $\mathrm{MCH}$ (ICR) or C57BL/6 strain to generate F2 (CxMxM) or F2 (CxMxB) mice. F2 (CxMxM) mice were bred with either the $\mathrm{MCH}$ (ICR) or C57BL/6 strain to generate F3 (CxMxMxM) or (CxMxMxB) mice, respectively.

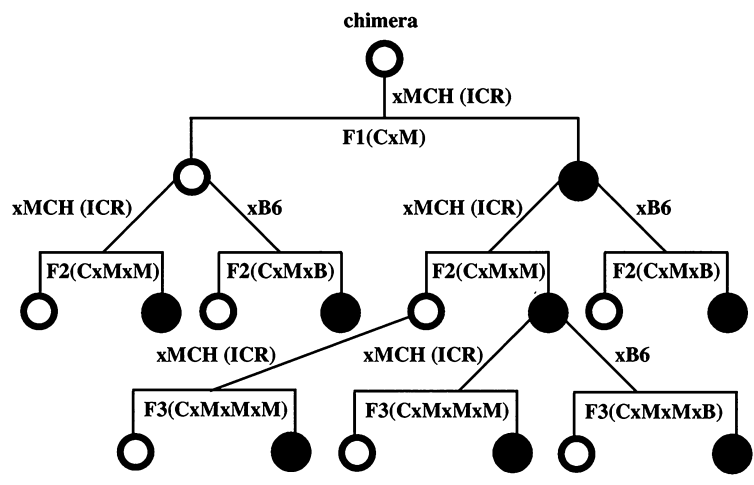

b
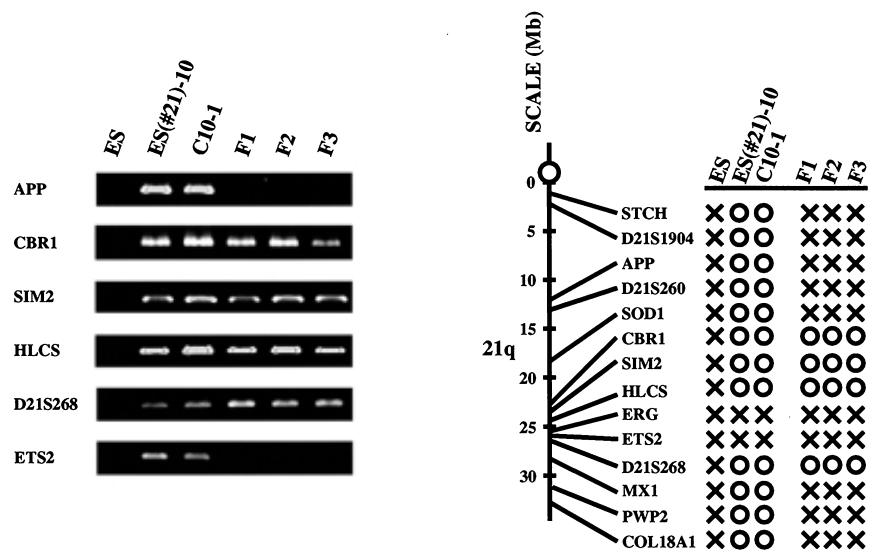

Fig. 1a-c. Characterization of transchromal (Tc) mice carrying human chromosome 21 fragment (hCF21). a A scheme for the breeding procedure. A chimeric female mouse was bred with an MCH (ICR) male mouse. F1 mice were bred with either $\mathrm{MCH}$ (ICR) or C57BL/6 mice. F2 (CxMxM) mice were bred with either MCH(ICR) or C57BL/6 mice. b Detection of hChr21 genomic DNA in tail fibroblasts of a chimeric mouse and Tc mice. Polymerase chain reaction (PCR) analyses were performed using 14 primers for the human chromosome 21-specific STS markers and genes. Significant results of six markers are presented. ES (TT2F), ES (\#21)-10, and chimera10-1 were used as negative and positive controls for PCR, respectively. c Summary of the retained region of hCF21 in Tc mice. $o$, Presence; $x$, absence. Randomly selected two F1 Tc mice, five F2 Tc mice, and five F3 Tc mice showed the same pattern of marker retention, irrespective of genetic background

Integrity and retention of hCF21 in F1, F2, and F3 mice

ERG and ETS2 markers had been deleted during the process of transfer of the hChr 21 to ES cells. PCR analyses of genomic DNA from tail fibroblasts showed that other regions of the hCF21 that had been retained in the ES cells were further deleted during transmission from the chimeric mouse to the F1 mice (Shinohara et al. 2001). The transmitted fragments in the F1 to F3 mice included at least CBR1, SIM2, HLCS, and D21S268 markers (Fig. 1b,c). The size of the hCF was estimated to be about $5 \mathrm{Mb}$ from the 
Fig. 2a-d. Detection of hCF21 in cells from Tc mice to examine the influences of genetic background on the stability of $\mathrm{hCF}$ in Tc mice. Fluorescence in situ hybridization (FISH) with human COT-1 DNA to a metaphase chromosomes isolated from tail fibroblasts, and $\mathbf{b}$ nuclei isolated from cerebral cortex of an F2 mouse. On metaphase spreads a, a single human chromosome (red signal with arrowhead) was detected in addition to mouse chromosomes (blue). c,d The retention of hCF21 in Tc mice with different genetic backgrounds. Fibroblasts prepared from tail samples from 4- to 10 -week-old Tc mice and cerebral cortex from 20- to 80-week-old Tc mice in F1-F3 generations with different combinations of genetic background were used for metaphase and interphase preparations, respectively. FISH was performed using the human COT-1 probe and 50 metaphase or interphase spreads were scored to determine the retention and integrity of hCF21. C, Chimeric mouse; $M, \mathrm{MCH}$ (ICR); B, C57BL/6. Closed and open circles indicate male and female, respectively. Statistical analysis was performed using the $t$-test. * $P<0.05$; \#, $P<0.001$
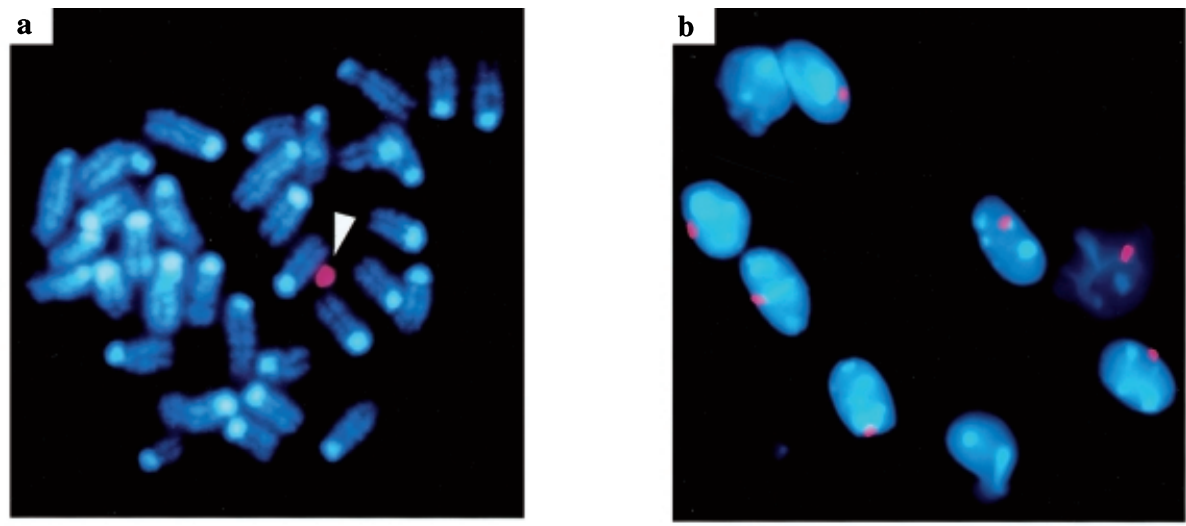

C

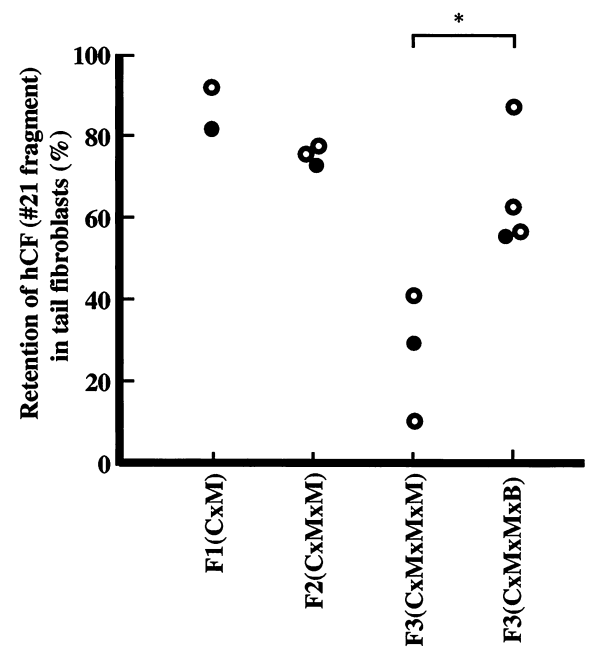

d

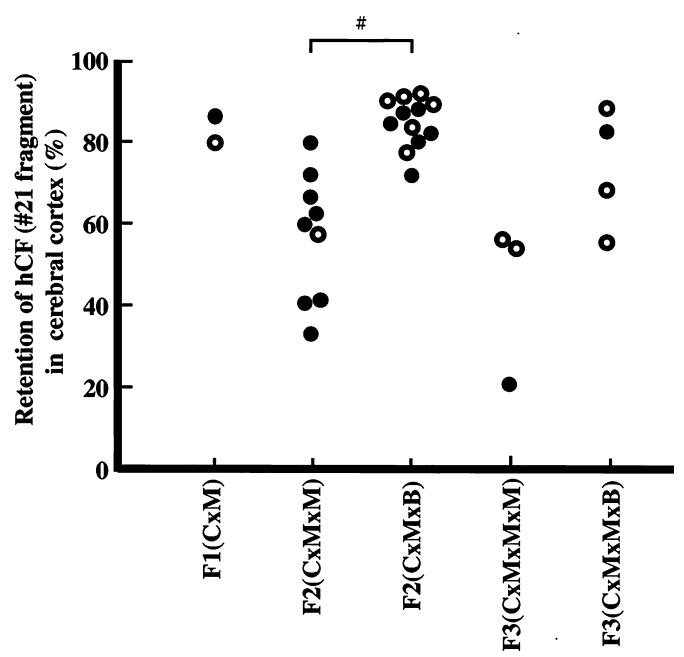

physical distance of the markers at both ends. The contents of the hChr 21 retained in Tc mice were consistent through F1 to F3 generations. No difference was observed in retained region of the hChr 21 between Tc mice with the two genetic backgrounds. FISH analyses of chromosomes in tail fibroblasts showed the independence of the hCF in mouse background (Fig. 2a), suggesting that no deletion or translocation had occurred during this breeding test.

FISH analysis of tail fibroblast was performed to test the stability of hCF21 in the different genetic backgrounds (Fig. $2 \mathrm{c})$. The hCF21 was retained in $55 \%-87 \%$ of tail fibroblasts in F3 (CxMxMxB) mice, but only in $10 \%-41 \%$ in $\mathrm{F} 3$ $(\mathrm{CxMxMxM})$ mice. This difference was statistically significant $(P<0.05)$. The previous study showed that the retention rate of hCF14 and hCF2 was higher in brain than in other tissues and that retention in tail fibroblasts in vivo may decrease corresponding to cell division (Shinohara et al. 2000). Thus, the retention of hCF21 in the cerebral cortex was also tested (Fig. 2b,d). The hCF21 was retained in $72 \%-92 \%$ of cerebral cortex cells in $\mathrm{F} 2(\mathrm{CxMxB})$ mice, whereas it was retained in $33 \%-80 \%$ in $\mathrm{F} 2(\mathrm{CxMxM})$ mice. This difference was statistically significant $(P<0.001)$. In the progeny of F2 (CxMxM) mice, the hCF21 was relatively more stable $(P=0.09958)$ in $\mathrm{F} 3(\mathrm{CxMxMxB})$ mice $(55 \%-$
$88 \%)$ than in $\mathrm{F} 3(\mathrm{CxMxMxM})$ mice $(21 \%-56 \%)$, which is consistent with our previous study with the hCF14 and the hCF2 (Shinohara et al. 2000). These results indicate that the competence to maintain hCF in these different strains has a genetic basis. The C57BL/6 background is preferable for stable maintenance of hCF. The Tc mice retained a subset of genes located in the so-called "Down syndrome critical region" in human chromosome 21q22 (Antonarakis 1998). Thus, these mice should allow us to investigate the genes related to or responsible for Ts 21 phenotypes. We are examining behavioral and pathological abnormalities of these Tc mice.

Stability of transferred human chromosome fragment in cultured cells and in mice has been assessed (Shen et al. 1997; Tomizuka et al. 2000; Shinohara et al. 2000). It was reported that the minichromosome derived from human chromosome $\mathrm{Y}$ was poorly maintained in the mouse ES cells, but was stabilized by rearrangement and incorporation of mouse centromere sequences (Shen et al. 1997). By contrast with the $\mathrm{Y}$ chromosome, the hCF21 described here was structurally stable and present as an independent chromosome without translocations in the Tc mice. Based on the results, we are currently constructing a human artificial chromosome using the centromeric region of hCF21 by a 
combination of Cre/loxP-mediated chromosome translocation and telomere-directed chromosome truncation in homologous recombination-proficient chicken DT40 cells (Kuroiwa et al. 1998, 2000).

Acknowledgments We thank C. Okita, H. Tsujii, and H. Hoshiya, Tottori University, for technical assistance. This work was supported by grants from the Japan Science and Technology Corporation, Japan and Research Project of Drug Safety Agency, and the Ministry of Education, Science, Sports and Culture of Japan.

\section{References}

Antonarakis SE (1998) 10 Years of genomics, chromosome 21, and Down syndrome. Genomics 51:1-16

Inoue J, Mitsuya K, Maegawa S, Kugoh H, Kadota M, Okamura D, Shinohara T, Nishihara S, Takehara S, Yamauchi K, Schulz TC, Oshimura M (2001) Construction of 700 human/mouse A9 monochromosomal hybrids and analysis of imprinted genes on human chromosome 6. J Hum Genet 46:137-145

Kola I, Hertzog PJ (1997) Animal models in the study of the biological function of genes on human chromosome 21 and their role in the pathophysiology of Down syndrome. Hum Mol Genet 6:1713-1727

Korenberg JR, Chen XN, Schipper R, Sun Z, Gonsky R, Gerwehr S, Carpenter N, Daumer C, Dignan P, Disteche C, Graham JM Jr, Hudgins L, McGillivray B, Miyazaki K, Ogasawara N, Park JP,
Pagon R, Pueschel S, Sack G, Say B, Schuffenhauer S, Soukup S, Yamanaka T (1994) Down syndrome phenotypes: the consequences of chromosomal imbalance. Proc Natl Acad Sci USA 24:4997-5001 Kuroiwa Y, Shinohara T, Notsu T, Tomizuka K, Yoshida H, Takeda S, Oshimura M, Ishida I (1998) Efficient modification of a human chromosome by telomere-directed truncation in high homologous recombination-proficient chicken DT40 cells. Nucleic Acids Res 26:3447-3448

Kuroiwa Y, Tomizuka K, Shinohara T, Kazuki Y, Yoshida H, Ohguma A, Yamamoto T, Tanaka S, Oshimura M, Ishida I (2000) Manipulation of human minichromosomes to carry greater than megabasesized chromosome inserts. Nat Biotechnol 18:1086-1090

Shen MH, Yang J, Loupart ML, Smith A, Brown W (1997) Human mini-chromosomes in mouse embryonal stem cells. Hum Mol Genet 6:1375-1382

Shinohara T, Tomizuka K, Takehara S, Yamauchi K, Katoh M, Ohguma A, Ishida I, Oshimura M (2000) Stability of transferred human chromosome fragments in cultured cells and in mice. Chromosome Res 8:713-725

Shinohara T, Tomizuka K, Miyabara S, Takehara S, Kazuki Y, Inoue J, Katoh M, Nakane H, Iino A, Ohguma A, Ikegami S, Inokuchi K, Ishida I, Reeves RH, Oshimura M (2001) Mice containing a human chromosome 21 model behavioral impairment and cardiac anomalies of Down syndrome. Hum Mol Genet 10:1163-1175

Tomizuka K, Shinohara T, Yoshimda H, Uejima H, Ohguma A, Tanaka S, Sato K, Oshimura M, Ishida I (2000) Double transchromosomic mice: maintenance of two individual human chromosome fragments containing immunoglobulin heavy and kappa loci and expression of fully human antibodies. Proc Natl Acad Sci USA 97:722-727 